\title{
Modification of the transmission spectrum of the "semiconductor-dielectric" photonic crystal in an external magnetic field
}

\author{
Svetlana V. Eliseeva*, Irina V. Fedorova, Dmitry I. Sementsov \\ Department of High Technology Physics and Engineering, \\ Ulyanovsk State University, Lev Tolstoy 42, 432700, Ulyanovsk, Russian Federation \\ *corresponding author, E-mail: eliseeva-sv@yandex.ru
}

\begin{abstract}
In this paper, some features of electromagnetic transmission of the waves of "semiconductor dielectric" periodic Bragg structure with a finite number of periods have been investigated. In the absence of absorption, for a structure with p-type semiconductor layers, we have analyzed the dependences on the external magnetic field of photon spectrum and transmission coefficient spectrum. It has been shown that with an increasing magnetic field, there is a significant narrowing of bandwidth and broadening of band gaps, as well as formation of new band gaps in the resonance region. The boundaries of all forbidden and allowed bands are shifted to higher frequencies with the increase of the angle of radiation incidence.
\end{abstract}

\section{Introduction}

In recent years, a remarkably increased interest of the researchers in the photonic crystal structures (PCS) is explained not only by the presence of photonic band gaps $[1,2,3]$ in their frequency spectrum, but also by the possibility to have an effective control of their optical characteristics. The configuration of the band spectrum, transmission and reflection coefficients are determined by the dispersion of eigen waves, their type and propagation direction against the axis of structure symmetry and controlling field $[4,5]$.

One of the most examined materials is one-dimensional PCS representing planar layered periodic structures whose properties can be effectively controlled $[6,7]$. In the structures with layers of doped semiconductor, the wave characteristics depend strongly on the magnitude and direction of the external magnetic field towards the direction of wave propagation and the structures periodicity axis. The features of propagation of electromagnetic waves in such a structure are largely associated with a gyrotropy of layers of doped semiconductor that can appear both in microwaves and optical ranges $[8,9,10,11,12,13]$.

In this paper, for the structure with a finite number of alternating layers of a p-type semiconductor and an isotropic dielectric, we have obtained the dispersion equation and the expression for energy transmission coefficient for the TM wave. Based on the numerical analysis, we have examined the dependence of the above characteristics on the external magnetic field and the waves incidence angle on the structure.
It is shown that with the growth of the field, there is a qualitative change in the character of transmission spectra. This is expressed not only in modification of the spectral position and width of the transmission and non transmission areas, but also in creation of new transmission ranges. With the increase in the angle of the radiation incidence on the structure, the boundaries of all forbidden bands are shifted at different speeds to the region of higher frequencies.

\section{Material relations}

Let us consider a one-dimensional PCS with a finite number of periods. The structure of one period $d$ includes a semiconductor layer with the thickness $d_{1}$ and a dielectric layer with the thickness $d_{2}$. The structures periodicity axis coincides with the $O Z$ axis direction, and the external magnetic field $H_{0}$ lies within the layers plane and coincides with the $O Y$ axis. The wave vector of the incident wave lies in the $X Z$ plane and makes an angle with the $O Z$ axis $\psi_{0}$ (Fig. 1). Dielectric layers will be assumed to be isotropic with a scalar dielectric constant (DC or permittivity) $\varepsilon_{d}$. The magnetic field leads to the anisotropy of the semiconductors optical properties, making its DC a tensor. The structures gyrotropic properties are due to the presence of non-diagonal antisymmetric components of the DC tensor $\varepsilon_{s}$. If we neglect the relaxation processes, the non-zero components of the tensor have the following form $[3,7]$ :

$$
\begin{array}{r}
\varepsilon_{x x}=\varepsilon_{z z}=\varepsilon, \quad \varepsilon_{y y}=\varepsilon_{l}\left(1-\frac{\omega_{p}^{2}}{\omega^{2}}\right), \\
\varepsilon_{z x}=\varepsilon_{x z}=i \varepsilon_{a}, \\
\varepsilon=\varepsilon_{l}\left(1-\frac{\omega_{p}^{2}}{\omega^{2}-\omega_{H}^{2}}\right), \quad \varepsilon_{a}=\frac{\varepsilon_{l} \omega_{p}^{2} \omega_{H}}{\omega\left(\omega^{2}-\omega_{H}^{2}\right)} .
\end{array}
$$

where $\varepsilon_{l}$ is the lattice part of DC semiconductor, $\omega_{p}=$ $\sqrt{4 \pi e^{2} n_{0} / m^{*} \varepsilon_{l}}$ and $\omega_{H}=e H_{0} / m^{*} c$ is the plasma and cyclotron frequency. Here, $m^{*}$ - is the effective mass of charge carriers in the semiconductor under study, $e=$ $4.8 \cdot 10^{-10}$ units of CGSE and $n_{0}$ - is the charge module and the carrier concentration. The plasma frequency plays the role of the "cutoff" frequency, below which the electromagnetic wave can not propagate in an unmagnetized (when $H_{0}=0$ ) solid-state plasma. In this case, at $\omega<\omega_{p}$ of the plasma, DC is negative and the refractive index of the plasma becomes an imaginary quantity. The cyclotron frequency at $H_{0} \neq 0$ is resonant, since when $\omega$ tends to $\omega_{H}$ 


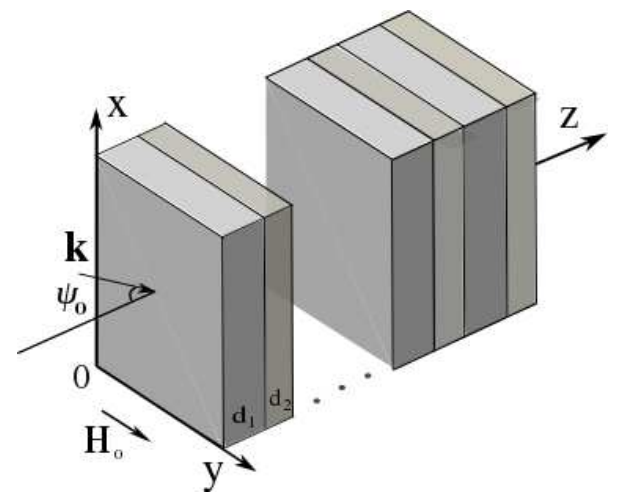

Figure 1: Geometry of the problem.

the moduli of the quantities $\varepsilon$ and $\varepsilon_{a}$ grow abruptly. The quantities $\varepsilon$ and $\varepsilon_{a}$ tendency to infinity is a consequence of the fact that dissipative processes driven by collisions of the charge carriers with the lattice were not taken into account while obtaining (1). In this case, the currents of conductivity can be neglected in Maxwell's equations in comparison with displacement currents. Allowance for dissipation leads to the complexity of the components of the DC tensor and to the finiteness of the real and imaginary parts of the magnetoactive plasma.

When the wave propagates perpendicular to the external magnetic field, each of the structures layers can have two eigen waves with orthogonal polarization - TE and $T M$. The wave characteristics of the first waves type do not depend on external magnetic field and are not considered by us. The behavior of $T M$ waves depends strongly on the magnitude of the applied field.Thus, the features of the semiconductors high-frequency behavior are largely related to the frequency and field dependence of the effective semiconductor permittivity:

$$
\varepsilon_{\perp}=\varepsilon-\frac{\varepsilon_{a}^{2}}{\varepsilon},
$$

\section{Dispersion in the fine-stratified medium}

The wave field of $T M$ wave contains components $\left\{E_{x}, H_{y}, E_{z}\right\}$. We assume the dependence of these components on time and longitudinal coordinates (along the axis $O X)$ to be proportional to the factor $\exp \left[i\left(\omega t-k_{x} x\right)\right]$, where the planar component of the wave vector is the same in all the layers of the structure, i.e.

$$
k_{x}=k_{0} \sqrt{\varepsilon_{0}} \sin \psi_{0}=k_{0} \sqrt{\varepsilon_{\perp}} \sin \psi_{1}=k_{0} \sqrt{\varepsilon_{d}} \sin \psi_{2} .
$$

Here, $-k_{0}=\omega / c, c$ is the light speed in vacuum, $\varepsilon_{\perp}=\varepsilon-\varepsilon_{a}^{2} / \varepsilon$ is the semiconductors effective permittivity in the magnetic field, $\varepsilon_{0}$ is the DC of the media in which the PCS is located, $\psi_{0}$ is the angle of incidence on the structure, measured from the $O Z$ axis, $\psi_{1}$ and $\psi_{2}$ are the angles between the normal and the wave vector in the layers of the semiconductor and the dielectric, respectively. The normal components of the wave vector in each of these layers are

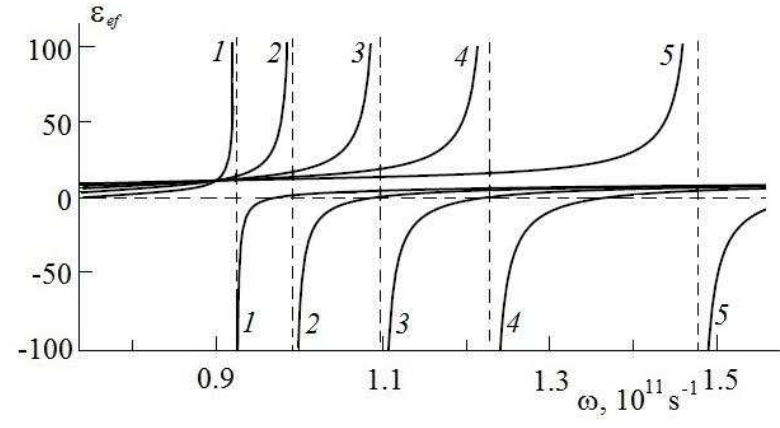

Figure 2: The frequency dependence of $\varepsilon_{e f}$ at $H_{0}=$ $0,0.5,1.5,2,3 \mathrm{kOe}$ (curves $1-5$ ) in the absence of absorption.

defined by the expressions:

$$
k_{z 1}=\left(k_{0}^{2} \varepsilon_{\perp}-k_{x}^{2}\right)^{1 / 2}, \quad k_{z 2}=\left(k_{0}^{2} \varepsilon_{d}-k_{x}^{2}\right)^{1 / 2} .
$$

The solution to the boundary problem together with periodicity conditions (for the tangential component of the wave field) results in the following dispersion relation:

$$
\begin{array}{r}
\cos (K d)=C_{1} C_{2}- \\
\frac{\varepsilon_{\perp} \varepsilon_{d}}{2 k_{z 1} k_{z 2}}\left[\left(\frac{k_{z 1}}{\varepsilon_{\perp}}\right)^{2}+\left(\frac{k_{z 2}}{\varepsilon_{d}}\right)^{2}+k_{x}^{2}\left(\frac{\varepsilon_{a}}{\varepsilon \varepsilon_{\perp}}\right)^{2}\right] S_{1} S_{2},
\end{array}
$$

where $K$ is the Bloch wave number, and the following notations have been introduced: $C_{j}=\cos \left(k_{z j} d_{j}\right), S_{j}=$ $\sin \left(k_{z j} d_{j}\right), j=1,2$

For a fine-stratified structure, when the condition $\lambda \gg$ $d_{1}+d_{2}$ (or $K d=2 \pi d / \lambda<<1$ ) is satisfied, it is possible to use a long-wave approximation, the dispersion relation takes the form:

$$
K=k_{0} \sqrt{\varepsilon_{e f}\left(\theta, \psi_{0}\right)} .
$$

Here, we have introduced an effective permittivity of a fine-stratified medium at an arbitrary angle of incidence of the TM wave on the structure:

$\varepsilon_{e f}\left(\theta, \psi_{0}\right)=\varepsilon_{e f}(\theta)-\left(\left(\varepsilon_{d}+\theta \varepsilon_{\perp}\right) \frac{\varepsilon_{d}+\varepsilon_{\perp}}{\varepsilon_{d}}-\varepsilon_{d} \frac{\varepsilon_{a}^{2}}{\varepsilon^{2}}\right) \frac{\sin ^{2} \psi_{0}}{\varepsilon_{\perp}(1+\theta)^{2}}$,

where the parameter $\theta=d_{1} / d_{2}$. Equation (6) determines the spectrum of the eigen waves, which can propagate in a fine-layered periodic structure for a given external field and wave propagation direction. When the wave propagates along the periodicity axis of the structure (at $\psi_{0}=0$ ), the expression (6) reduces to the form $K=k_{0} \sqrt{\varepsilon_{e f}(\theta)}$, where

$$
\varepsilon_{e f}(\theta)=\frac{\varepsilon_{d}+\theta \varepsilon_{\perp}}{(1+\theta)} .
$$

Fig. 2 shows frequency dependences of the effective semiconductor permittivity structure of the value corresponding to the values of the external magnetic field $H_{0}=$ 

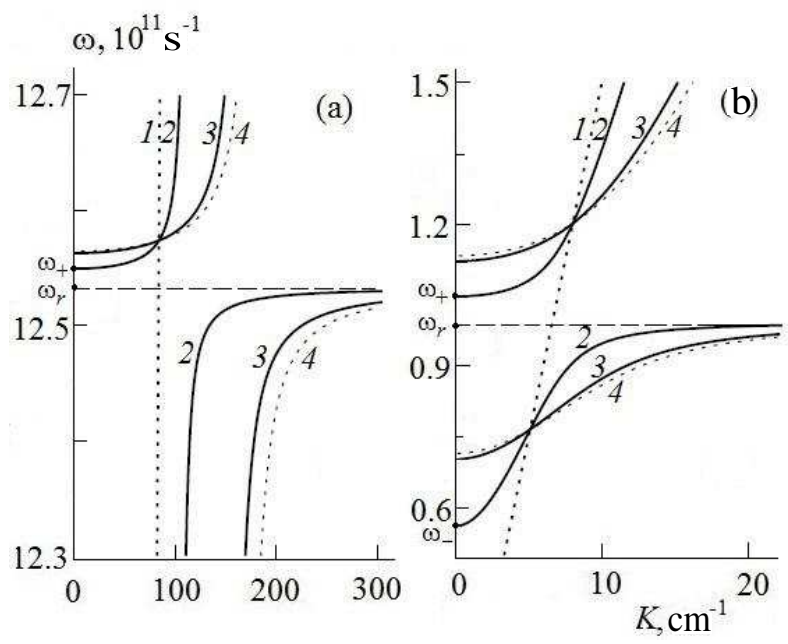

Figure 3: The dispersion relations $\omega(K)$ for a fine-layered structure in which the semiconductor layers can be of the $n$ - or $p$-type $(\mathrm{a}, \mathrm{b})$ at $H_{0}=1 k O e$ in the absence of absorption and parameter values $\theta=0,0.25,4, \infty$ (curves $1-4)$.

$(0.5,1,1.5,2,2.8) \mathrm{kOe}$ (curves 1-5). To create them, let us assume that the thicknesses of the layers in the structure period are equal $\left(d_{1}=d_{2}, \theta=1\right)$. The material $\mathrm{SiO}_{2}$ with DC $\varepsilon_{d}=4$ is selected as a dielectric layer. For the semiconductor layers ( $I n S b$-type), we used the following parameter values [7]: $\varepsilon_{l}=17.8$, the carriers effective mass is $m^{*}=0.42 m_{0}$ (where $m_{0}$ is free electron mass), the concentration is $n_{0}=1.9 \cdot 10^{13} \mathrm{~cm}^{-3}$, the plasma frequency $\omega_{p}=9 \cdot 10^{10} s^{-1}$. At this value of the field, the cyclotron resonance frequency for the holes is defined by the expression $\omega_{H}=4.2 \cdot 10^{7} H_{0} s^{-1}$. An effective permittivity is experiencing dramatic changes in the magnitude and the sign near the resonant frequency of the structure, which is defined in case of equal layer thicknesses in the period by the expression:

$$
\omega_{r e s} \simeq \sqrt{\omega_{H}^{2}+\gamma \omega_{p}\left(\omega_{p}+\omega_{H}\right)},
$$

where the parameter $\gamma=\varepsilon\left(\varepsilon_{l}+\varepsilon_{d}\right)^{-1}$. With the increase of the controlling magnetic field, there is a highfrequency shift of the resonance curves. Fig. 3 shows the dispersion relations $\omega(K)$ for a wave propagating along the axis of periodicity of a fine-layered structure in which the semiconductor layers can be of the $n$ - or $p$-type $(\mathrm{a}, \mathrm{b})$. To create them, the following values of the layers parameters were used: for dielectric layers $\varepsilon_{d}=4\left(\mathrm{SiO}_{2}\right)$, for semiconductor layers $\varepsilon_{l}=17.8$ ( $\left.\operatorname{In} S b\right)$; for $n$-type semiconductor, the effective mass of carriers $m^{*}=0.014 m_{0}$ and their carrier concentration $n_{0}=0.65 \cdot 10^{12} \mathrm{~cm}^{-3}$; for $p$-type $m^{*}=0.42 m_{0}$ and $n_{0}=1.9 \cdot 10^{13} \mathrm{~cm}^{-3}, m_{0}$ is mass of a free electron. At the chosen carrier concentrations, the plasma frequency for both types of semiconductor is the same and equal to $\omega_{p}=9 \cdot 10^{10} \mathrm{~s}^{-1}$. The above dependences were obtained with no absorption assumption in the layers of the semiconductor $(\nu=0)$ at an external magnetic field $H_{0}=1 k O e$ and parameter values $\theta=$ $0,0.25,4, \infty$ (curves 1-4). For a given value of the field, the cyclotron resonance frequency for electrons and holes is determined by the expressions $\omega_{H n}=12.5 \cdot 10^{8} H_{0} s^{-1}$ and $\omega_{H p}=0.42 \cdot 10^{8} H_{0} s^{-1}$. The presence of an external field leads to the splitting of the dispersion curves and to the appearance in the spectrum of high-frequency and lowfrequency branches. The frequencies of these branches at $K \rightarrow 0$ are given by the expressions:

$$
\omega_{ \pm}=\sqrt{\frac{q \pm \sqrt{q^{2}-4\left(\varepsilon_{d}+\theta \varepsilon_{l}\right) \varepsilon_{l} \theta \omega_{p}^{4}}}{2\left(\varepsilon_{d}+\theta \varepsilon_{l}\right)}},
$$

where $q=\left(\varepsilon_{d}+\theta \varepsilon_{l}\right) \omega_{H}^{2}+\left(\varepsilon_{d}+2 \theta \varepsilon_{l}\right) \omega_{p}^{2}$. We note that in the case under consideration $H_{0} \neq 0$, in the absence of absorption, the dispersion curves of each of the branches intersect at one point with frequencies At $K \rightarrow \infty$ the low-frequency branch tends asymptotically to the frequency $\omega_{r}=\sqrt{\omega_{H}^{2}+\omega_{p}^{2}}$. As a result of the splitting of the dispersion curves, a frequency gap is formed in the spectrum in which the wave is damped. The width of the gap $\Delta=\omega_{+}-\omega_{r}$ depends considerably on the ratio of the thicknesses of the layers and the external magnetic field. In the region $\omega_{r}<\omega<\omega_{+}$, the wave in the PCS is damped and the transmission coefficient can take anomalously small values. Near resonant frequencies $\omega_{r}^{(n)} \approx 12.532 \cdot 10^{11} s^{-1}$ and $\omega_{r}^{(p)} \approx 0.994 \cdot 10^{11} s^{-1}$ in the spectra, there is a structure of condensing alternating allowed and forbidden narrow bands.

\section{Dispersion relations and transmission spectra}

The analysis of the relationship (6) shows, - that in the general case of periodic structure, when $\lambda \sim d$, the dispersion dependences $K(\omega)$ are alternating the forbidden and allowed frequency bands. The band gaps reflect the structures opacity, i.e. an effective reflection of the wave with the reflection coefficient of a unit value, the allowed bands reflect the structures transparency. The transmission coefficient of the PCS consisting of $n$ periods can be expressed through the components of the transfer matrix of the structure $\hat{M}$. According to the Abeles theorem [7, 14], this matrix is determined by the $n$-th degree of the transfer matrix of one period $\hat{M}=(\hat{m})^{n}$. Its components have the form of

$$
\begin{array}{r}
M_{j j}=m_{j j} \frac{\sin (n K d)}{\sin (K d)}-\frac{\sin ((n-1) K d)}{\sin (K d)}, \\
M_{j, 3-j}=m_{j, 3-j} \frac{\sin (n K d)}{\sin (K d)},
\end{array}
$$

where $j=1,2$, and the elements of the matrix $\hat{m}$ are given in the Appendix. At the same time, the wave amplitude coefficient of the wave transmission by the structure takes the form [9]:

$$
t=\frac{2 k_{0} k_{z a} \exp \left(-i k_{z b} n d\right)}{k_{0} k_{z a} M_{11}+k_{0} k_{z b} M_{22}-k_{z a} k_{z b} M_{12}-k_{0}^{2} M_{21}} .
$$



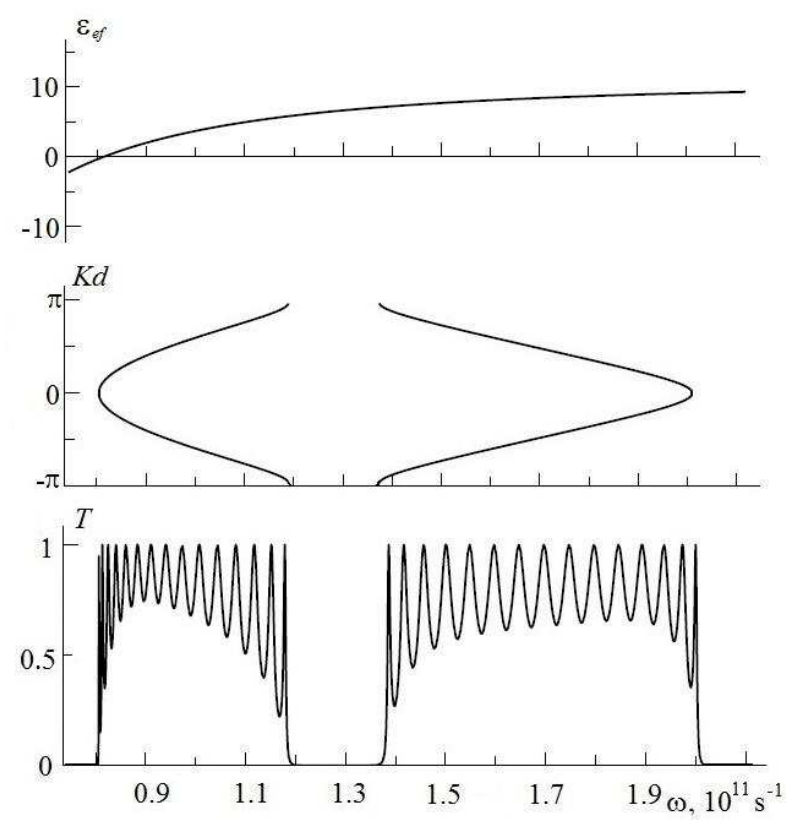

Figure 4: The frequency dependence of effective DC of the structure $\varepsilon_{e f}$, the Bloch wave number $K$ and the transmission coefficient $T$ at $H_{0}=0$ and in the absence of absorption.

For the numerical analysis of the PCS bandwidth, we use the energy transmission coefficient $T=\left(n_{b} / n_{a}\right)|t|^{2}=$ $|t|^{2}$, where we take into account that the refractive indices of the media bordering the PCS are the same: $n_{a}=n_{b}=$ $\sqrt{\varepsilon_{0}}$.

Let us first consider the wave properties of the considered PCS in the absence of an external magnetic field. Fig. 4 shows the frequency dependence of three variables an effective permittivity of the structure $\varepsilon_{e f}$, the Bloch wave number $K$ and the transmission coefficient $T$, that are obtained for the case of wave propagation along the periodicity axis $\left(\psi_{0}=0\right.$ and $\left.k_{x}=0\right)$. It can be seen that at the frequency

$$
\omega_{r e s}(0)=\omega_{p} \sqrt{\varepsilon_{l} /\left(\varepsilon_{l}+\varepsilon_{d}\right)} \simeq 8.1 \cdot 10^{10} \mathrm{~s}^{-1} .
$$

there is a change of the sign of the effective permittivity. In the area of $\omega<\omega_{\text {res }}(0)$, the permittivity $\varepsilon_{e f}$ is negative, so this area is forbidden on the dispersion relation. The spectrum of the transmission coefficient $T(\omega)$ represents an oscillating function with alternating transmission and nontransmission bands. For the area $\omega>\omega_{\text {res }}(0)$, the figure shows the first two areas of transparency and the first Bragg band gap. The two following figures shows evolution of the dispersion relations, which is observed with an increasing magnetic field in the case of the wave propagation along the axis of periodicity.

Fig. 5 shows the dependences $K(\omega)$ within the field intervals $H_{0}=(20-370) O e$. It is seen that in the case of small fields, at which the resonance frequency is permitted in the first zone, the dispersion relation change occurs only in this area. The dispersion curves type in the second

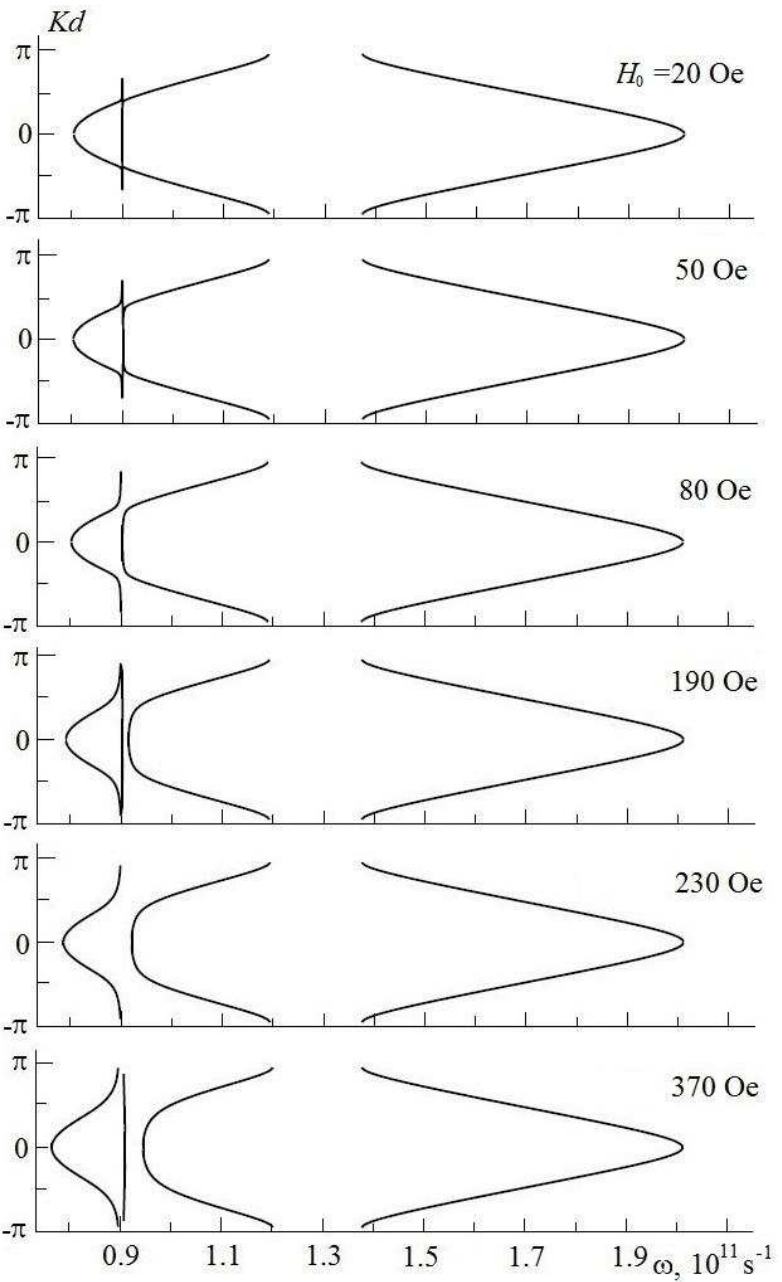

Figure 5: The photon spectrum of PCS $K(\omega)$ at $H_{0}=$ 20, 50, 80, 190, 370 Oe, $\left(d_{1}=d_{2}=0.15 \mathrm{~cm}\right)$.

allowed band and the forbidden bands width remain practically unchanged in these fields. The character of changes in the dependence $K(\omega)$ in the first zone consists in the following: first, at the resonance frequency, there is a split of the zone into two subzones $\left(H_{0}=50 \mathrm{Oe}\right)$. With a further increase of the field, there is an expansion the resulting forbidden area $\left(H_{0}>80 \mathrm{Oe}\right)$, and appearance at the resonant frequency of a new narrow allowed band $\left(H_{0} \geq 190 \mathrm{Oe}\right)$.

Fig. 6 shows the evolution of the dependence $K(\omega)$ with a further increase of the field within the values range $H_{0}=(0.5-2.8) k O e$. In these fields, the spectrum modification occurs not only in the area of the first, but also the second allowed band (in the absence of the field). There is a significant expansion of the forbidden bands and narrowing of the second allowed band, as well as appearance of additional bands at the field values $H_{0}=(1.5,2.8) \mathrm{kOe}$. Such a change in the dispersion spectrum in a magnetic field should affect the reflection and transmission spectra for the structure under consideration.

Fig. 7 shows the spectra of the transmission coefficient $T(\omega)$ for the considered interval of the magnetic field. According to the dependence $K(\omega)$, the increasing 

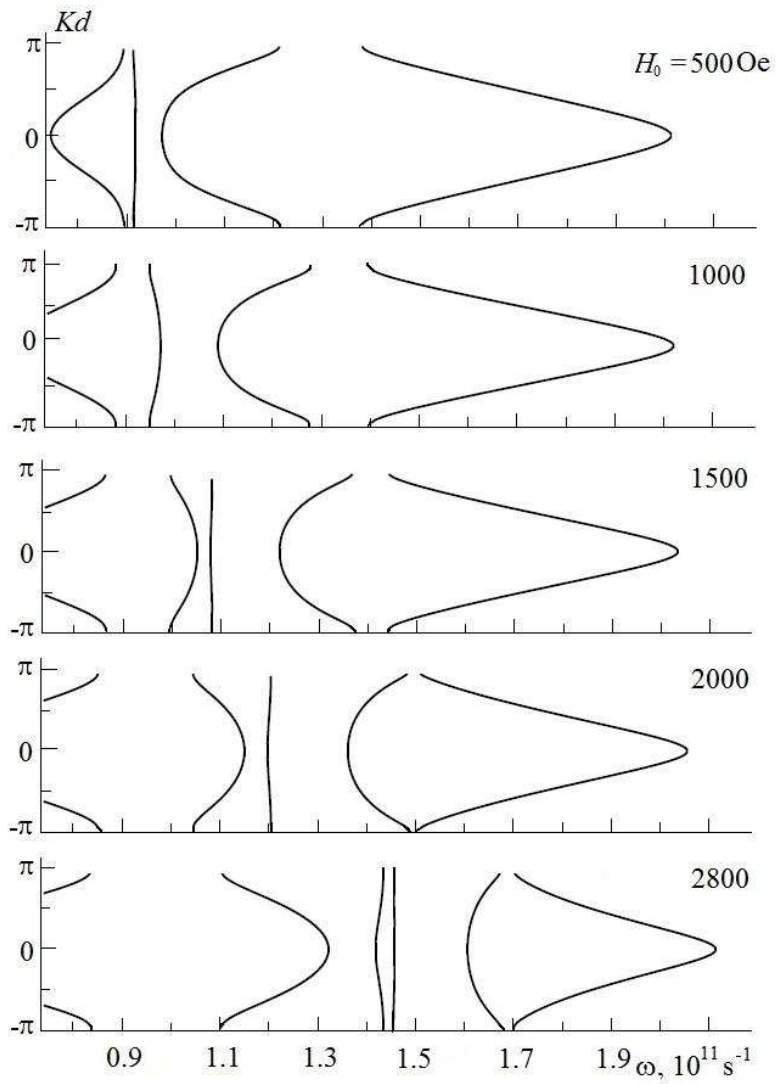

Figure 6: The photon spectrum of PCS $K(\omega)$ at $H_{0}=$ $0.5,1.0,1.5,2.0,2.8 \mathrm{kOe},\left(d_{1}=d_{2}=0.15 \mathrm{~cm}\right)$.

field triggers extension of the opacity bands with the maximum reflectivity capacity of the structure. In these stopbands, there might appear narrow minibands of the structures transparency near the resonance frequency.

Fig. 8 shows the diagram $T\left(\omega, \psi_{0}\right)$, illustrating the dependence of the transmission coefficient on the frequency and incident radiation angle. These diagrams were obtained for the magnetic field values $H_{0}=(0,0.5,2.0) k O e$. It can be seen that with an increasing external magnetic field, there is a significant broadening of the forbidden zones and their shift to the region of high frequencies. Near the resonant frequency, a narrow permitted miniband appears in the band gap, with the frequency range practically independent on the angle of incidence on the structure. The position and the width of the allowed and forbidden bands that are close to the resonance are weakly dependent on the incidence angle. However, with an increase in the angle $\psi_{0}$, the boundaries of all forbidden and allowed bands are shifted at a different speed to higher frequencies area.

\section{Conclusions}

The paper investigates controllability of the transmittivity of "semiconductor-dielectric" PCS by an external magnetic field in the high-frequency range (in area $\omega \simeq 10^{11} \mathrm{~s}^{-1}$ ). On the basis of numerical analysis of the dispersion relation
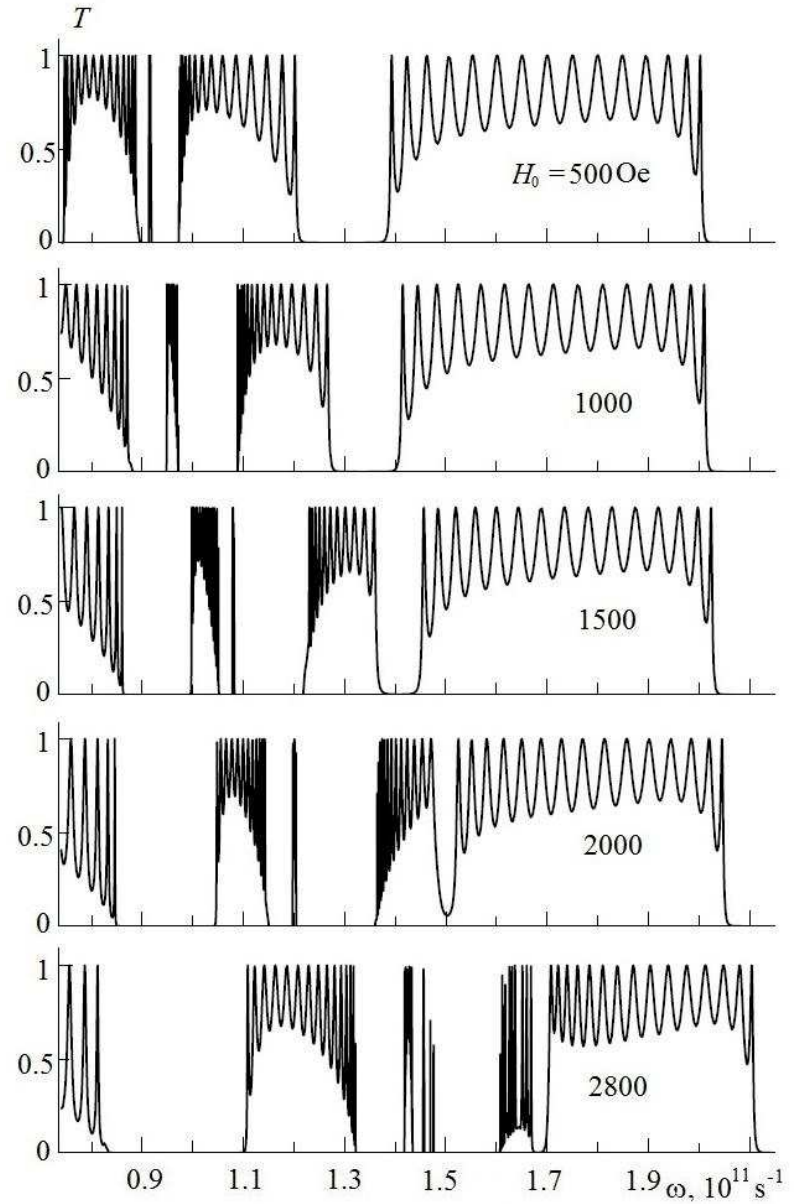

Figure 7: The spectra of the transmission coefficient $T(\omega)$ at $H_{0}=0.5,1.0,1.5,2.0,2.8 \mathrm{kOe}$.

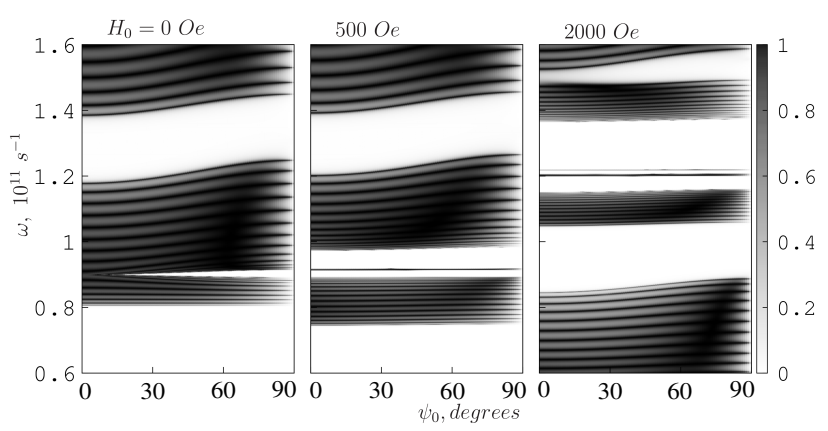

Figure 8: The map of the distribution of the transmission coefficient versus the frequency $\omega$ and incident radiation angle $\psi_{0}$.

for TM wave in the periodic structure and energy transmission coefficient, we have investigated the above characteristics dependence on the magnitude of the external magnetic field. To better understand the features of the magnetic field influence on the structures photon spectrum, layers of a semiconductor of the hole type (with parameters $p-I n S b$ ) are considered in the absence of absorption. This approx- 
imation is completely acceptable in the investigated frequency range and the adopted impurity concentrations. The dispersion dependences of the TM wave for a structure with semiconductor layers of the $n$ - and $p$-type have been analyzed for different ratios of a semiconductor and a dielectric layers thicknesses and an external magnetic field. The features of these characteristics have been revealed in the region of cyclotron resonance, which is observed in a relatively narrow frequency interval for the given field value. It is shown that the spectral position of the transmission and non-transmission areas of radiation of the PCS with a finite number of periods significantly depends on the fields value. The results analysis has shown that by changing the magnetic field, one can effectively control the width of the opacity zones and the transmission coefficient value in this range of frequencies. Thus, the fields presence causes narrowing of the available transmission areas and emergence of new zones in comparison to the PCS spectrum in the absence of the magnetic field. This results in an increase in the width of the forbidden bands. By increasing the angle of incidence on the structure, the boundaries of the forbidden and allowed bands are shifted at a different speed to higher frequencies.

We also note that the use of metal layers in the PCS to induce a gyrotropy due to their high conductivity and, correspondingly, the small depth of the skin layer $(\delta \leq \mu \mathrm{m})$ is ineffective. The waves of this range penetrate the metal layer only slightly and are almost completely reflected.

\section{Appendix}

Below, there are transmission matrices for some individual layers of a semiconductor and a dielectric making the structures period:

$$
\begin{gathered}
\hat{m}_{1}=\left(\begin{array}{cc}
C_{1}-\frac{k_{x} \varepsilon_{a}}{k_{z 1} \varepsilon} S_{1} & i \frac{k_{0} \varepsilon_{\perp}}{k_{z 1}} S_{1} \\
\left(i \frac{k_{z 1}}{k_{0} \varepsilon_{\perp}}+i \frac{k_{x}^{2} \varepsilon_{a}^{2}}{k_{z 1} \varepsilon^{2}}\right) S_{1} & C_{1}+\frac{k_{x} \varepsilon_{a}}{k_{z 1} \varepsilon} S_{1}
\end{array}\right), \\
\hat{m}_{2}=\left(\begin{array}{cc}
C_{2} & i \frac{k_{0} \varepsilon_{d}}{k_{z 2}} S_{2} \\
i \frac{k_{z 2}}{k_{0} \varepsilon_{d}} S_{2} & C_{2}
\end{array}\right)
\end{gathered}
$$

as well as transmission matrix components for one pe$\operatorname{riod} \hat{m}=\hat{m}_{1} \cdot \hat{m}_{2}$ :

$$
\begin{array}{r}
m_{11}=\left[C_{1}-\frac{k_{x} \varepsilon_{a}}{k_{z 1} \varepsilon} S_{1}\right] C_{2}-\frac{k_{z 2} \varepsilon_{\perp}}{k_{z 1} \varepsilon_{d}} S_{1} S_{2}, \\
m_{12}=-\frac{k_{0} \varepsilon_{d}}{i k_{z 2}}\left[C_{1}-\frac{k_{x} \varepsilon_{a}}{k_{z 1} \varepsilon} S_{1}\right] S_{2}-\frac{k_{0} \varepsilon_{\perp}}{i k_{z 1}} S_{1} C_{2}, \\
m_{21}=\left(i \frac{k_{z 1}}{k_{0} \varepsilon_{\perp}}+i \frac{k_{x}^{2} \varepsilon_{a}^{2}}{k_{z 1} \varepsilon^{2}}\right) S_{1} C_{2}+\frac{i k_{z 2}}{k_{0} \varepsilon_{d}}\left[C_{1}+\frac{k_{x} \varepsilon_{a}}{k_{z 1} \varepsilon} S_{1}\right] S_{2}, \\
m_{22}=\frac{i k_{0} \varepsilon_{d}}{k_{z 2}}\left(i \frac{k_{z 1}}{k_{0} \varepsilon_{\perp}}+i \frac{k_{x}^{2} \varepsilon_{a}^{2}}{k_{z 1} \varepsilon^{2}}\right) S_{1} S_{2}+\left[C_{1}-\frac{k_{x} \varepsilon_{a}}{k_{z 1} \varepsilon} S_{1}\right] C_{2} .
\end{array}
$$

where the following notations have been introduced:

$$
C_{j}=\cos \left(k_{z j} d_{j}\right), S_{j}=\sin \left(k_{z j} d_{j}\right), j=1,2
$$

\section{Acknowledgement}

This work was partially supported by the Ministry of Edu-

cation and Science of the Russian Federation (Contract No. 14.Z50.31.0015 and No.3.6825.2017/BCh).

\section{References}

[1] M. Inoue,M. Levy, A. V. Baryshev, Magnetophotonics: From theory to applications, Springer Science \& Business Media, 2013.

[2] S. A. Maier, Plasmonics: fundamentals and applications, Springer Science \& Business Media, 2007.

[3] K. Sakoda, Optical properties of photonic crystals, Springer Science \& Business Media 80: 2004.

[4] S. G. Johnson, J. D. Joannopoulos, Photonic crystals: the road from theory to practice, Springer Science \& Business Media, 2001.

[5] E. Yablonovitch, Photonics: One-way road for light, Nature 461: 744-745, 2009.

[6] A. Yariv, P. Yeh, Optical waves in crystals, Wiley, New York, 1984.

[7] F. G. Bass, A. A. Bulgakov, A. P. Tetervov, Highfrequency properties of semiconductors with superlattices, Moscow Izdatel Nauka, 1989.

[8] S. Erokhin, L. Deych, A. Lisyansky, A. Granovsky, Magneto-optical effects in excitonic one-dimensional structures, Solid State Phenomena 152: 503-507, 2009.

[9] V. Kumar, B. Suthar, J. Malik, A. Kumar, K. S. Singh, T. Singh, A. Bhargva, Defect mode properties and origin in one dimensional photonic crystal, Photonics and Optoelectronics, 2013.

[10] S. Eliseeva, D. Sementsov, Optical spectra of onedimensional defect photonic crystals, Optics and Spectroscopy 109: 729-737, 2010.

[11] V. I. Fesenko, I. V. Fedorin, V. R. Tuz, Dispersion regions overlapping for bulk and surface polaritons in a magnetic-semiconductor superlattice, Optics letters 41: 2093-2096, 2016.

[12] A. I. Ignatov, A. M. Merzlikin, M. Levy, A. P. Vinogradov, Formation of degenerate band gaps in layered systems, Materials 5: 1055-1083, 2012.

[13] Y. S. Dadoenkova, N. Dadoenkova, D. Korobko, I. Zolotovskii, D. Sementsov, I. Lyubchanskii, Tunnelling of frequency-modulated wavepackets in photonic crystals with amplification, Journal of Optics 18: 015102, 2015. 
[14] M. Born, E. Wolf, Principles of optics: electromagnetic theory of propagation, interference and diffraction of light, Elsevier, 1980. 